\title{
EDITORIAL
}

\section{Therapie von Sexualstraftätern}

\author{
Hans-Ludwig Kröber
}

Online publiziert: 22. Dezember 2012

(C) Springer-Verlag Berlin Heidelberg 2012

Therapie ist das neue Zaubermittel der Rechtspolitik. Seitdem das Bundesverfassungsgericht sich der Straßburger Vorgabe unterworfen hat, dass Maßregeln der Besserung und Sicherung nur dann keine Strafe sind, wenn sie der Behandlung einer psychischen Störung dienen, figuriert die Therapie quasi als Beweis für die psychische Gestörtheit der Insassen. Der unverbesserliche Rückfalltäter wird nicht mehr wegen seiner Gefährlichkeit, sondern zum Zwecke der Behandlung seiner psychischen Störung weggesperrt. Delinquenz ist (im juristischen Diskurs) in Gestalt der „antisozialen“ oder „dissozialen Persönlichkeitsstörung“ zu einer weiteren psychischen Krankheit geworden, und so genannte Hirnforscher liefern willig vermeintliche Beweise, dass Delinquenz eine Hirnkrankheit sei. Alle gesunden Menschen sind von Natur aus gesetzestreu; wir ahnten es und gehören dazu.

Die Sexualstraftäter standen schon immer im Verdacht, psychisch gestört zu sein, weil ihr Verhalten ja ,nicht normal" ist. Früher war auch Homosexualität strafbar, und tatsächlich gab es psychiatrische Behandlungsangebote zur Minderung oder Unterdrückung der Homosexualität, wenn nicht gar zur Umwandlung der Orientierung. Man sieht: Therapie soll eine Anpassung des Einzelnen an soziale Normen erreichen; sie beweist nicht eo ipso, dass eine psychische Störung vorliegt.

Andererseits gibt es insbesondere im Bereich der sexuellen Nötigung und der Vergewaltigung eine Vielzahl von Tätern, angefangen bei Jugendlichen, die eine „,normale“ sexuelle Orientierung haben und auch keine ungewöhnlichen Praktiken begehren, aber nach dem Prinzip der sofor-

Prof. Dr. med. H.-L. Kröber $(\bowtie)$

Institut für Forensische Psychiatrie,

Charité - Universitätsmedizin Berlin,

Oranienburger Str. 285, 13437 Berlin, Deutschland

E-Mail: hans-ludwig.kroeber@charite.de tigen und notfalls rücksichtslosen Bedürfnisbefriedigung verfahren. Nicht jeder von diesen wird gestört sein und einer Therapie bedürfen; Strafe wirkt manchmal sehr nachhaltig.

In diesem Heft mit dem Schwerpunkt „Sexualstraftätertherapie" befassen wir uns mit den Fragen: Wer benötigt Therapie? Wie kann eine solche Therapie strukturiert sein, welche Ziele soll sie anstreben? Wie wirksam sind solche Therapien?

Wir beginnen mit einem Beitrag über das Konstrukt der „Hypersexualität“ - gibt es das überhaupt, ist es eine Verhaltensvariante oder tatsächlich eine behandlungswürdige Störung, und welche sozialen Gefahren für andere sind damit verbunden? Wie es scheint, ist zu viel tatsächlich ungesund und sozial riskant.

Es folgt dann ein Beitrag über das „Risk-need-responsivity“ (RNR)-Modell, wonach die Tätertherapie sich an der Gefährlichkeit der jeweiligen Person orientieren soll, dessen kriminogene Faktoren adressieren und dabei seine Verstehensmöglichkeiten berücksichtigen soll. Dieses Modell liegt den meisten gruppentherapeutischen Maßnahmen im Maßregelvollzug und Sozialtherapie zugrunde; die Autorinnen benennen in ihrer Übersicht auch die Schwachpunkte dieses Modells, nämlich die eher defensive Orientierung der Vermeidung von Risikofaktoren, bei unzureichender Ausrichtung auf Wünsche und Bedürfnisse des Verurteilten, was zu häufigen Therapieabbrüchen gerade bei jenen mit hohem Rückfallrisiko führt. Die Bedürfnisse und Lebensziele des Insassen sind wiederum die Ausgangspunkte des „Good Lives Model“, über das dann von Franqué und Briken einen kurzen Überblick geben. Über dieses wichtige therapeutische Konzept werden wir sicher auch weiterhin berichten.

Nach diesen Übersichten werden spezielle Aspekte diskutiert. Kann man Sexualstraftäter mit „objektiven“, sprich technischen Messverfahren hinsichtlich devianter 
oder gewaltbereiter Struktur identifizieren, wie uns dies ja nicht zuletzt in den Medien angeboten wird? Die Hamburger Arbeitsgruppe ist hier ausgesprochen skeptisch. Dafür verdeutlicht sie in der Diskussion von männlicher „Ritterlichkeit", dass es bei der Behandlung von Sexualstraftätern bisweilen eher um Erziehung zu modernen Gender-Konzepten als um Psychotherapie gehen mag, um das Abtrainieren von Macho-Attitüden nicht nur in der Muskelshirt-Liga, sondern auch in der Smoking Lounge. Ein eigener Beitrag erinnert daran, dass die Behandlung von Sexualstraftätern in Haft und Maßregelvollzug von einem enormen Machtgefälle geprägt wird, das die Gefahr birgt, dass der Klient den besseren Einsichten der Therapeuten unterworfen statt in seinen prosozialen Fähigkeiten gefördert wird; Transparenz und Fairness werden ganz konkret zu wichtigen Faktoren für Gelingen oder Scheitern. Da nicht leicht zu sagen ist, wann eine solche Therapie gelungen ist, drucken wir die Übersetzung der - noch nicht vollständig evaluierten - Therapiebeurteilungsskala (TRS-10) ab, weil sie uns allemal hilfreich zu sein scheint, nicht zuletzt für unsere Diskussion, was wir denn erreichen wollen.

Es folgt ein Beitrag über die Veränderung kriminogener Faktoren bei Sexual- und Gewaltstraftätern in Sozialtherapie und Regelvollzug, der abermals verdeutlicht, wie schwer es ist, die Effekte der institutionellen Behandlung zu erfassen. Welche harten Prä-post-Daten gibt es, mit denen relevante Veränderungen durch eine mehrjährige Behandlung abgebildet werden könnten? Der Beitrag vermehrt die Skepsis über das Dogma, dass kriminogene Einstellungen zu Straftaten führen und veränderte Einstellungen zur Rückfallfreiheit. Die Schlucht zwischen Einstellung und einstellungskonformer Handlung ist methodisch noch nicht hinreichend überbrückt. (So bleiben die meisten feindseligen Einstellungen gottlob tatenlos.) Zudem schafft Therapie wohl keine Löschung von unguten Einstellungen, sondern ein Nebeneinander unterschiedlicher Werthaltungen, die je nach dem sozialen Kontext aktivierbar sind. Vielleicht gab es dieses Nebeneinander von prosozialen und dissozialen Konzepten schon zum Tatzeitpunkt; der Delinquent hatte halt die ungünstige Option gezogen. Wenn aber die Interaktion von Einstellungen und sozialem Lebensraum entscheidend ist, spricht viel für die Ausweitung ambulanter Interventionen. Im letzten Beitrag erläutern Häßler et al. die Möglichkeiten und Grenzen der elektronischen Aufenthaltsüberwachung insbesondere für die Rückfallprävention.

Hans-Ludwig Kröber 